\title{
Understanding Environmental Influence in an Open-Ended Evolutionary Algorithm
}

\author{
Andreas Steyven ${ }^{\star \star}$, Emma Hart, and Ben Paechter \\ School of Computing, Edinburgh Napier University, \\ 10 Colinton Road, Edinburgh, Scotland, United Kingdom \\ \{a.steyven, e.hart, b.paechter\}@napier.ac.uk
}

\begin{abstract}
It is well known that in open-ended evolution, the nature of the environment plays in key role in directing evolution. However, in Evolutionary Robotics, it is often unclear exactly how parameterisation of a given environment might influence the emergence of particular behaviours. We consider environments in which the total amount of energy is parameterised by availability and value, and use surface plots to explore the relationship between those environment parameters and emergent behaviour using a variant of a well-known distributed evolutionary algorithm (mEDEA). Analysis of the resulting landscape show that it is crucial for a researcher to select appropriate parameterisations in order that the environment provides the right balance between facilitating survival and exerting sufficient pressure for new behaviours to emerge. To the best of our knowledge, this is the first time such an analysis has been undertaken.
\end{abstract}

Keywords: evolutionary robotics; parameter selection; environment-driven evolution; distributed online adaptation

\section{Introduction}

Due to technological advances in both hardware and software, the vision of sending swarms of robots into unchartered terrains to monitor and map environments is becoming much closer to being realised. This brings significant new challenges for evolutionary robotics, with the need for completely distributed evolutionary algorithms to evolve controllers that enable robots to survive for long-periods of time. The issue of survival is key if robots are to effectively accomplish any kind of task: user-driven tasks cannot even be achieved if the integrity of the swarm is compromised through lack of ability to survive.

A number of recent algorithms tackle this issue, notably mEDEA [1] and its variations e.g. EDEA $_{r f}$ [7] and MONEE [6 4]. However, the emerging behaviours arising from the interactions of an open-ended evolutionary algorithm with its environment are not well understood, perhaps in part due to the timeconsuming experimentation that needs to be done to conduct sweeps of the parameters that define the environment. It is common in optimisation to explore

\footnotetext{
** This is the author's version of the work. It is posted here for your personal use. Not for redistribution. The definitive Version was published in PPSN XIV 2016, http://dx.doi.org/10.1007/978-3-319-45823-6_86
} 
the relationship between algorithmic parameters and fitness. However, evolutionary robotics adds an additional dimension in that it is not only the algorithms parameters that change but also the environmental parameters.

Given that it is the environment that provides the pressure to adapt in a purely open-ended scenario, it is crucial to gain some understanding of these landscapes. Particularly in simulation, it is easy to arbitrarily select environmental parameters such as the number of available energy sources or their corresponding energy-values. However, arbitrary choices can inadvertently create landscapes which have a major influence on the evolution of behaviour. For example, assume a researcher wishes to investigate whether individual learning speeds up environment-driven evolution: if an environment is created that has too much energy available then it is unlikely to exert sufficient pressure for individual learning to be beneficial or even emerge. Quantifying 'too much' (or 'too little') is of course difficult. In order to address this, we conduct an analysis of an open-ended evolution algorithm operating in a variable environment. To the best of our knowledge, this is the first time this has been attempted.

Using an open-ended evolutionary algorithm, $\operatorname{mEDEA}_{r f}$ [7, we consider evolved behaviours in environments in which the total energy available is parameterised by two variables that determine the availability and value of energy pellets within in the environment. Using a 3-dimensional visualisation of the energy landscape for EDEA $_{r f}$ we show:

- the energy landscape contains three distinct regions: energy-poor, energyneutral and energy-rich, as well as a 'dead-zone' in which robots cannot survive

- the energy-rich region is relatively large compared to other regions but is very rugged

- that on the energy-neutral line, distinct behaviours evolve at different places along the line

We propose that the energy-neutral region provides the most obvious settings for conducting experimentation that aims to extend a robots ability to survive or accomplish tasks.

\section{Related Work}

The completely distributed evolutionary algorithm for open-ended evolution $m E D E A$ was first proposed in [1]. It was tested using a scenario in which environmental pressure forces robots to compete for limited resources in order to gain energy. The algorithm was demonstrated to be both efficient with regard to providing distributed evolutionary adaptation in unknown environments, and robust to unpredicted changes in the environment. The basic algorithm has been extended in a number of ways.

Haasdijk et al 6] extended mEDEA so that in addition to surviving and operating reliably in an environment, a robot could also perform user-defined tasks. Their new framework MONEE (Multi-Objective aNd open-Ended Evolution algorithm) showed initially that task-driven behaviour can be promoted without 
compromising environmental adaptation. More recently, they investigated the trade-off between the survival and task-accomplishment that evolution must establish when the task is detrimental to survival, finding that task-based selection exerts a higher pressure than the environment. Fernandez et al 3 study the impact of adding explicit selection methods to the mEDEA algorithm in a task-driven scenario. They evaluate four selection methods that induce different intensities of selection pressure, using tasks that include obstacle avoidance and foraging, finding that higher selection pressure results in improved performances, especially in more challenging tasks. Hart [7] also extended mEDEA by including selection based on a fitness value that was calculated relative to those robots in the immediate vicinity, thus maintaining the decentralised nature of the algorithm, and additionally using this relative fitness value to control the frequency and range of broadcasting. Parameter tuning of algorithmic parameters to optimise algorithmic task-performance was investigated by [5]. However, to the best of our knowledge, no methodical investigation of environment parameter settings has been conducted: researchers tend to select arbitrary values or simply use those defined in previous papers.

\section{Algorithm Description}

Evolution of robot controllers is performed by the $\mathrm{mEDEA}_{r f}$, first introduced in 7. The algorithm is an extension of the original mEDEA algorithm of Bredeche et al [1] with the addition of an explicit fitness measure. This influences the spread of genomes through the population in order to increase survivability, thus ensuring the integrity of the swarm.

mEDEA $_{r f}$ utilises an agent driven by a control architecture whose parameters are defined by the currently active genome. The genome defines the weights of an Elman recurrent neural network (RNN) consisting of 16 sensory inputs, one bias node (feeding into the hidden layer) and 2 motor outputs (translational and rotational speeds). 8 ray-sensors are distributed around the robot's body. They detect the proximity to the nearest object and its type. The RNN has 1 hidden layer with 16 nodes, thus 322 weights are defined by the genome. This setup is adapted from [1. An overview of the algorithm is given in Algorithm 1 and reader is referred to [7] for more detail. In brief, for a fixed period, robots move according to their control algorithm, broadcasting their genome that is received and stored by any robot within range. At the end of this period, a robot uses roulette-wheel selection to choose a genome from its list of collected genomes according to a relative fitness value, and applies a variation operator. This takes the form of a Gaussian random mutation operator, inspired from Evolution Strategies. Robots that have not collected any genomes temporarily become inactive, thus reducing the population size.

Each robot estimates its fitness in terms of its ability to survive based on the balance between energy lost and energy gained, delta Energy $\left(\delta_{E}\right)$ : this term is initialised to 0 at $t=0$ (when the current genome was activated) and is decreased by 1 at each time-step, and increased by $E_{\text {token }}$ if it crosses an energy token. Given $\delta_{E}$, a robot calculates a fitness value which is relative to those robots in 
a range $r$ according to equation 1 , where $f_{i}^{\prime}$ is the relative fitness of robot $i$ at time $t$, mean $_{s u b_{i}}$ is the mean $\delta_{E}$ of the robots within the subpopulation defined by all robots in range $r$ of robot $i$, and $s d_{s u b_{i}}$ is the standard deviation of the $\delta_{E}$ of the subpopulation.

$$
f_{i}^{\prime}(t)=\frac{\delta_{i}(t)-\operatorname{mean}_{s u b_{i}}(t)}{s d_{s u b_{i}}(t)}
$$

Note that evolution is asynchronous, in keeping with the paradigm of a distributed algorithm without central control. If a robot runs out of energy and has an empty genome list, it remains stationary until it receives a new genome from a passing robot at which point it starts a new lifetime. Thus at any time-step, each robot potentially has a different 'age'.

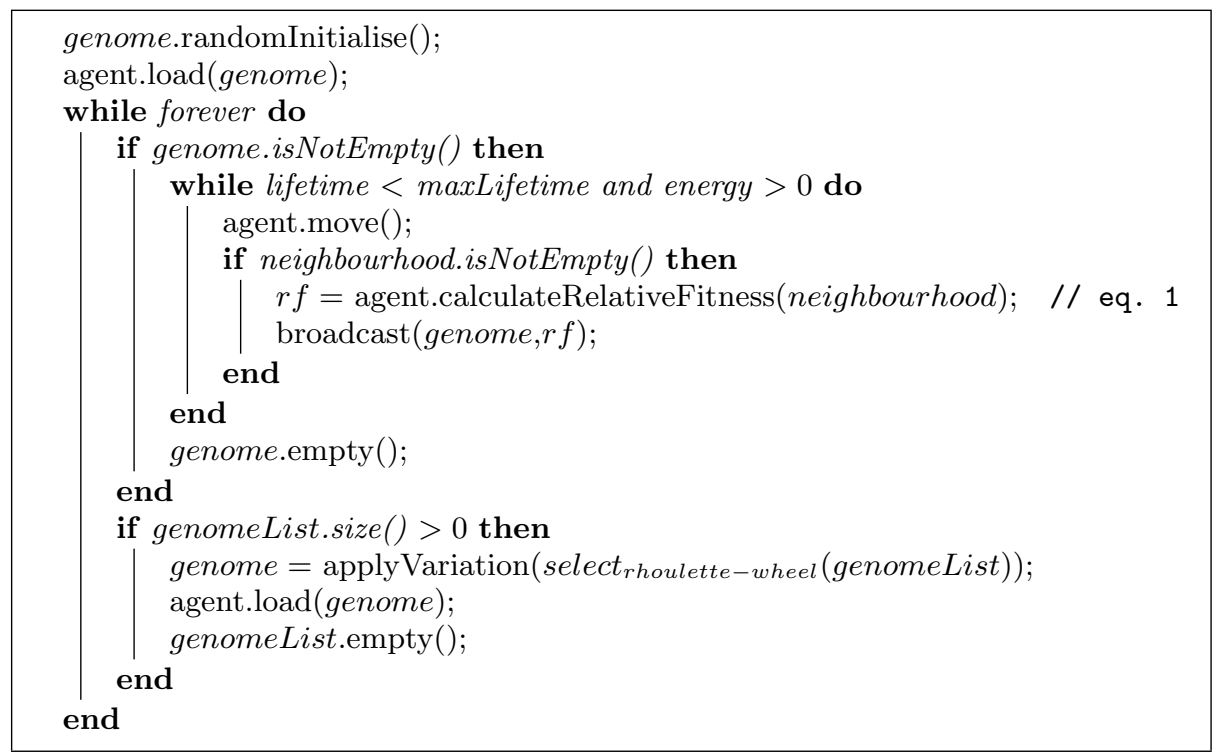

Algorithm 1: Pseudo code of our adapted version of the mEDEA algorithm based on vanilla mEDEA by Bredeche et al. [1]

\section{Method}

All experiments are conducted in simulation using Roborobo! by Bredeche et al. from [2]. A static environment is created, using an arena previously described in [364]. The robot cannot pass through the outer and inner walls, however, it is possible to broadcast through an obstacle. Energy tokens are randomly scattered in the environment. If a robot moves over a token, its energy is increased by an amount $E_{\text {token }}$. The energy token disappears when consumed and reappears after a fixed amount of time later at a different random location. Fixed parameters describing the simulation are given in table 1 . 
Energy is consumed in three ways. There is a fixed cost to 'living' of 0.5 units per timestep, regardless of whether the robot moves or not. A robot moving consumes an amount of energy $E_{m}$ that is related to its rotational speed $v_{\text {rot }}$, translational speed $v_{\text {trans }}$, and their respective maximum values $v_{\text {rot }}$ MAX and $v_{\text {trans }}$ MAX , and is given by

$$
E_{m}=\left(v_{\text {rot }} / v_{\text {rot }_{\mathrm{MAX}}}+v_{\text {trans }} / v_{\text {trans }_{\mathrm{MAX}}}\right) / 4
$$

Finally, a robot consumes energy when communicating. This is an important factor in the real-word but one that it is often overlooked in simulation models. The model used is exactly as described in [10, with an energy cost of $E_{R X}=$ 0.082 units for receiving and a cost of $E_{T X}(r)=0.075$ units for transmitting.

The goal of the experiments is to understand the energy landscape in terms of the median $\delta$ Energy of a robot in the population as a function of the two environmental parameters: count, the number of energy tokens available, and value, the energy value of each token. Table 1 shows the ranges of values considered for each parameter. Parameters are set before the beginning of the experiment and remain fixed throughout. Each experiments was repeated for 5 independent runs. This number is rather low for a noisy application of this type but was chosen to speed up computation due to the high number of experiments that had to be run in total.

Table 1: Simulation and Experimental Parameters for all experiments

\begin{tabular}{ll}
\hline Simulation parameters & \\
\hline Arena size & 1024 pixel by 1024 pixel \\
Max. robot lifetime & 2500 iterations \\
Token re-spawn time & 500 iterations \\
Sensor range & 196 pixel \\
\hline \hline Variable Parameters \\
\hline Number of robots \\
Number of tokens (count) & $50,75,100$ \\
Energy value per token $($ value $)$ & $0-1400$ (in steps of 50$)$ \\
\hline \hline Experimental parameters & \\
\hline Number of runs & 5 \\
Maximum iterations & $375000(=150 x 2500)$ \\
Start energy & 500 \\
Maximum range $r_{\max }$ & 128 \\
\hline
\end{tabular}

Data is gathered from the robots every 2500 iterations. Recall from section 3 that each robot chooses a new genome once it has depleted all its energy or reached the maximum lifetime, leading to asynchronous generation changes throughout the population. Hence, the data gathered at each interval represents a snapshot across robots of multiple ages and therefore does not necessarily capture the peak performance of each robot (i.e. it may include very 'young' robots). However, given that the goal of the experiment is to understand the interplay of the specific algorithm and environment under consideration, this is not a relevant factor. 


\section{Analysis}

Figure 1 shows three rotated 3-dimensional plots of surface obtained using 100 robots after 375,000 iterations. The $x$ and $y$ axes represent the count and value variables, while the $z$ axis represent the median $\delta_{E}$ of the robot population over the last 2500 iterations. The grey plane marks a value for $\delta_{E}$ of zero, at which point robots have an energy balance of zero, i.e. the same amount of energy as they started the experiment with. Three broad regions are noticeable: a large region in which the robots have positive $\delta_{E}$ (green and blue value above the grey plane), a region lying on the plane itself, and finally a region below the plane in which robots are spending more energy than they are collecting, i.e. $\delta_{E}<0$. In order to explore this in more detail, a 2-dimensional top-down projection is shown in figure 2 obtained from populations of 50, 75 and 100 robots, and is discussed in detail below.

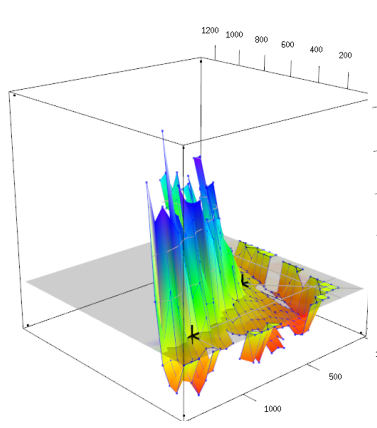

(a) rotated $90^{\circ}$ right

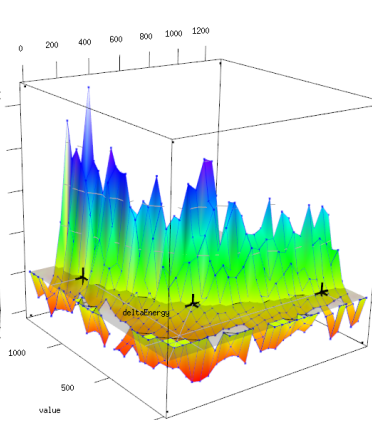

(b) centred

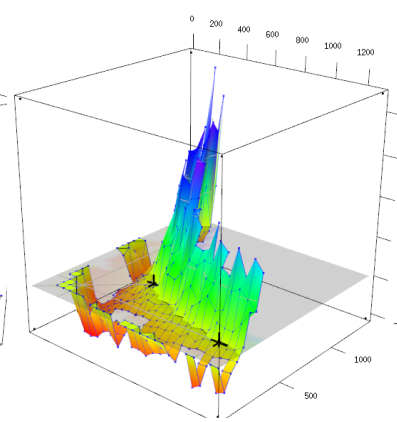

(c) rotated $90^{\circ}$ left

Fig. 1: View on the resulting surface from different angles. The figure was created by plotting the median $\delta_{E}$ of the last 2500 iterations of the experiment. The grey plane marks a value for $\delta_{E}$ of zero, at which point robots in an experiment have an energy balance of zero. In other words, the same amount of energy as they started the experiment with. A 3D model can be found at 9

\subsection{Different performance regions}

Figure 2 shows clearly that the landscape is defined by four different regions:

A) Dead Zone: In this region, the environment does not provide enough energy for the algorithm to evolve controller that can survive a full run. Low values for both parameters, count and value result in the extinction of the whole robot population within a few generations. The random genomes that the controllers are initialised with generally result in a random spinning behaviour, rather than movement. This random behaviour, combined with the lack of energy tokens in the immediate vicinity in which the robot is born, mean that robots cannot survive given its inability to move. 

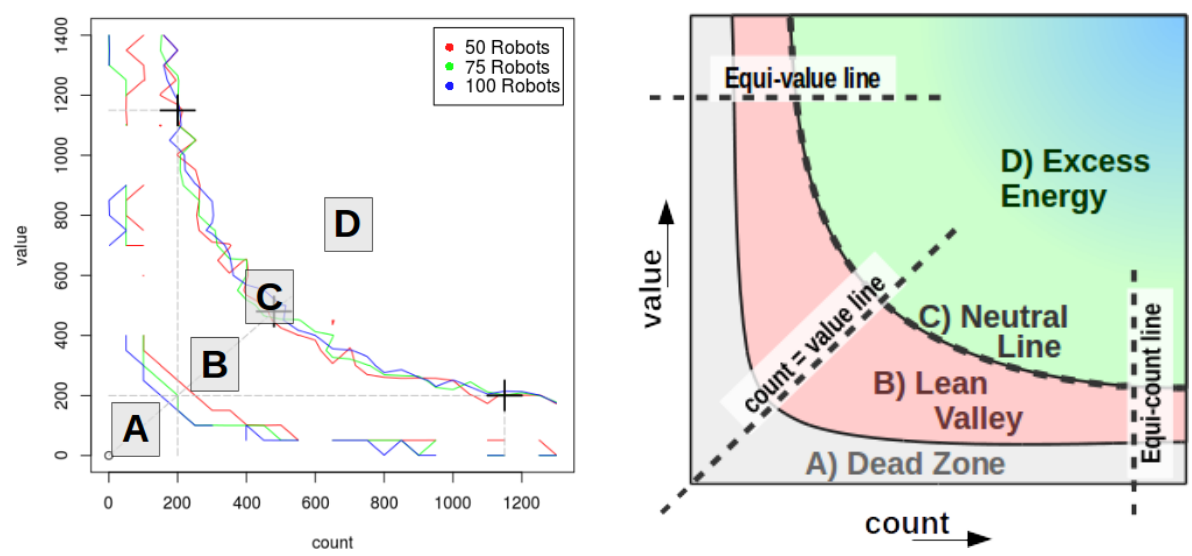

Fig. 2: Overview of landscape, as plot of the real data on the left and as a cartoon version on the right. 4 different regions are shown: A) Dead Zone, B) Lean Valley, C) Neutral Line, D) Excess Energy.

B) Lean Valley (negative $\delta_{E}$ ): This region starts at the edge of the dead zone that marks the point where there is just enough energy available that some robots survive until the end of the experiment, i.e. it marks the point where a robot has spent all its initial energy and started picking up tokens from the environment. Moving down towards the bottom of the valley, an increasing number of robots survive as there is more energy in environment, with the corollary that each robot has less total energy - the energy available is shared between more robots. The bottom of the valley marks the minimum $\delta_{E}$ that still enables survival. Moving upwards out of the valley on the other side, robots gradually get better in both harvesting energy from the environment and managing their residual energy as a result of evolving better strategies. For example, good strategies optimise movement, or avoid moving towards tokens in which there are other robots close by.

C) Neutral Line $\left(\delta_{E}=0\right)$ : This line marks the points in the environment where the environment provides exactly enough energy to enable a robot to maintain an energy balance of zero, i.e. the costs of moving and communicating are just balanced by energy harvested.

D) Excess Energy $\left(\delta_{E}>0\right)$ : In the final region, in which both cost and value are high, robots are able to locate more energy in the environment than is required to maintain their initial energy $E_{0}$, either due to the abundance of pucks or the high energy value of pucks.

\subsection{Environmental Influence on Behaviour}

In order to properly understand the evolved behaviours that lead to the landscapes just described, a more detailed analysis is required. Figure 3 examines 
pairings of (count, value) along the three dashed lines in 2 , i.e. equivalent-value (a-b), equivalent-count (c-d) and the diagonal in which count = value line (e-f). The figure shows boxplots of the $\delta_{E}$ values at specific pairings of (count, value) and the ratio of genome broadcasts made to unique genomes received over a lifetime. The latter quantity leads to insights into behaviour as it relates to the number of unique robots encountered by an individual robot: a robot will broadcast indiscriminately to any robot in its range but will only collect unique genomes. At the equivalent-count and equivalent-value lines, we fix the parameter count and value respectively, and successively increase the other parameter in steps of 50 .

5 points are shown. The first point on a) corresponds to a total energy $E_{t o t}$ that is the same as the first points on graphs (c) and (e) below it etc. ${ }^{1}$ For a specific value of $E_{t o t}$, then is clear that high value combined with low count leads to robots that have increased $\delta_{E}$ when compared to robots with high count but low value (graph (a) compared to graph (e)). Robots must therefore evolve behaviours that enable them to seek out the rare but high-value pucks. These robots also have high broadcast:genome ratios, suggesting the robots are frequently coming into contact with the same robots. A possible explanation lies in the fact that the robots appear travel in small groups, thus broadcasting continually to the same robots; the rare occurrence of pucks leads to many robots having to travel towards the same regions of the space. On the other hand, a high count leads to robots that receive more unique genomes than in the high value case: this is suggestive of a more random movement pattern that enables each robot to encounter many unique robots during its lifetime. In this case there is low selection pressure to evolve focused movement due to the abundance of pucks.

\subsection{Behaviours in the neutral region}

We propose that the energy neutral region is of greatest interest for researchers wishing to conduct research moving beyond genetic evolution of survival, for example using individual or social learning [8] or task-driven research [4]. In this region, on the one hand, robots are able to survive, while on the other, the environment does not over-provide, thus ensuring that there is scope for robots to learn novel behaviours. We further investigate three specific points within this region there is approximately the same amount of energy available in the environment (table 2). The table shows the median age increases with increasing count - it is easier to maintain sufficient energy to survive as availability increases. The lower median observed at low count reflects the fact that many robots do not survive long. The time to find a new unique genome (age:genome) is shortest at high count, reflecting frequent encounters with novel robots. Broadcast:genomes is highest at low count as observed in the previous section. All three configurations lead to the same energy balance of 0 , but diverse behaviours result in the gain in energy being offset by movement and broadcasting in each case.

${ }^{1}$ while this is exactly true for the first and third rows, in the middle row which represents equal count/value it is necessary to approximate 

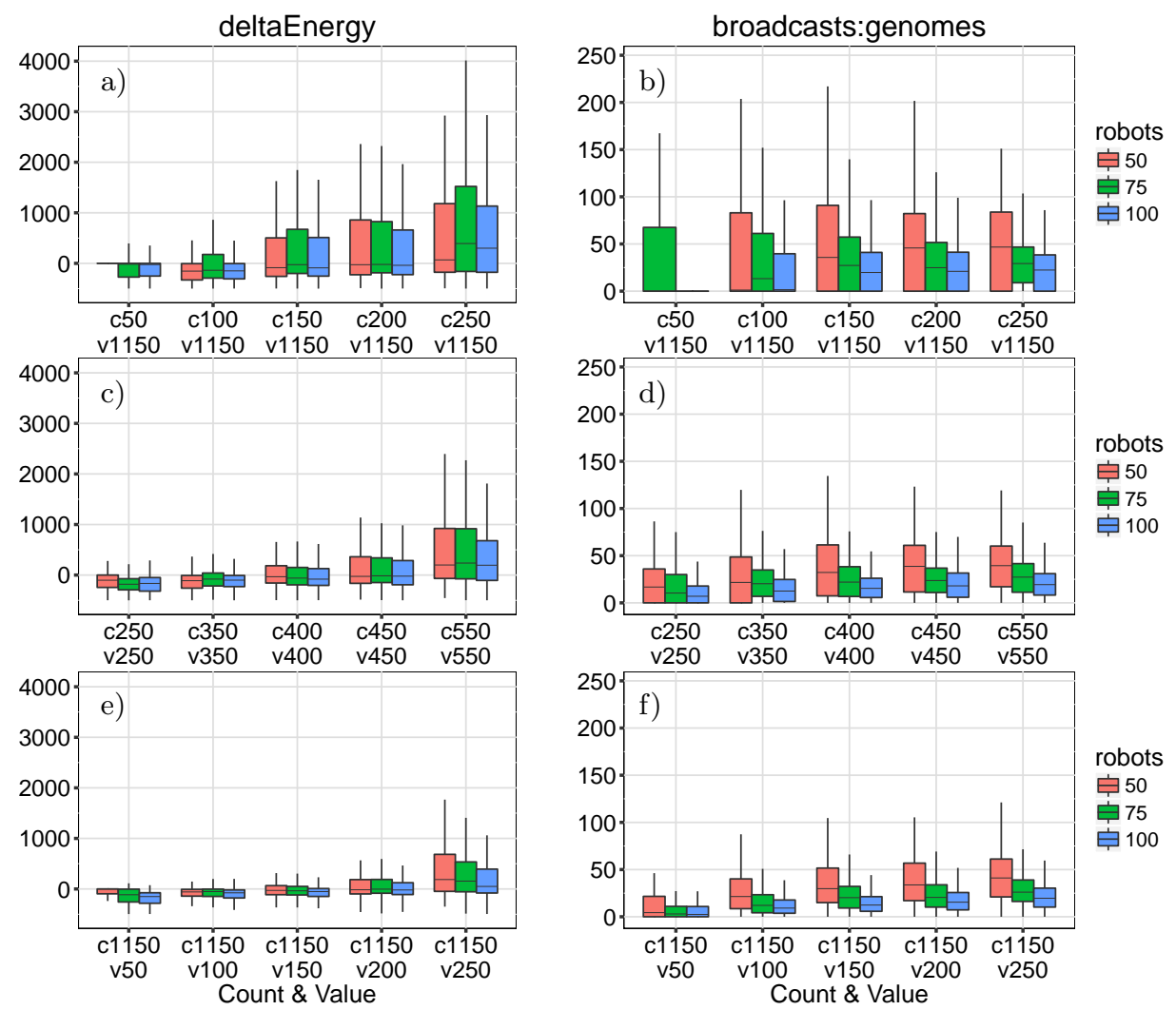

Fig. 3: Cuts through different parts of the landscape. Points towards different behaviours in terms of exploration. a-b) value $=1150$, vary count; $\mathrm{c}-\mathrm{d}$ ) count $=$ value; e-f) count $=1150$, vary value.

\section{Conclusion}

We have presented the first analysis of the fitness landscape (as a function of environmental parameter) that results from running an open-ended evolutionary algorithm $\left(\mathrm{mEDEA}_{r} f\right)$ in an environment that is parameterised by two values that control the distribution of energy in the environment. Adjusting the availability and value of energy pucks results in the evolution of a range of different behaviours. Rather than arbitrarily selecting parameters in which to study evolution, we suggest that it is vital to understand how these choices will direct evolution, by changing the selection pressure exerted by the environment.

Three distinct regions are observed in which the final energy balance can be negative, neutral, or positive. A fourth region is found in which robots cannot survive. We propose that the energy neutral region is a good region in which to undertake experiments. It provides an environment in which robots are able to survive, enabling experimentation, while at the same time, will reward new behaviours which are able to more efficiently harness energy from the environ- 
Table 2: Results obtained at three configurations within the neutral region

\begin{tabular}{|c|c|c|c|c|c|c|c|c|c|c|}
\hline & \multicolumn{9}{|c|}{ Robots } \\
\hline & & \multicolumn{3}{|c|}{50} & \multicolumn{3}{|c|}{75} & \multicolumn{3}{|c|}{100} \\
\hline Count & Value & Age & $\frac{\text { Age }}{\text { Genome }}$ & $\frac{\text { Brodcasts }}{\text { Genome }}$ & Age & $\frac{\text { Age }}{\text { Genome }}$ & $\frac{\text { Brodcasts }}{\text { Genome }}$ & Age & $\frac{\text { Age }}{\text { Genome }}$ & $\frac{\text { Brodcasts }}{\text { Genome }}$ \\
\hline 200 & 1150 & 7770 & 107.94 & 46.61 & 767.5 & 63.86 & 28.99 & $66^{7}$ & 49.97 & 21.18 \\
\hline 500 & 500 & 1026.5 & 86.12 & 39.11 & 1038.5 & 52.39 & 25.93 & 928.5 & 41.26 & 19.67 \\
\hline 1150 & 200 & 1173.5 & 79.83 & 33.43 & 1093 & 47.39 & 21.95 & 1059 & 36.52 & 15.49 \\
\hline
\end{tabular}

ment. It is clear that the environment plays a key role in influencing what kind of behaviours emerge, in that it is not the total amount of energy available that matters but also the manner in which it is spread. Future work should be aimed at understanding the landscape in more detail, and in particular, explaining the ruggedness of some regions.

\section{References}

1. Bredeche, N., Montanier, J.M.: Environment-driven embodied evolution in a population of autonomous agents. In: Schaefer, R., Cotta, C., Koodziej, J., Rudolph, G. (eds.) PPSN XI. vol. 6239, pp. 290-299. Springer Berlin Heidelberg (2010)

2. Bredeche, N., Montanier, J.M., Weel, B., Haasdijk, E.: Roborobo! a fast robot simulator for swarm and collective robotics. CoRR abs/1304.2 (4 2013)

3. Fernández Pérez, I., Boumaza, A., Charpillet, F.: Comparison of selection methods in on-line distributed evolutionary robotics. In: ALife'14. pp. 282-289. MIT Press (2014)

4. Haasdijk, E.: Combining conflicting environmental and task requirements in evolutionary robotics. In: 2015 IEEE 9th International Conference on Self-Adaptive and Self-Organizing Systems. pp. 131-137. IEEE (9 2015)

5. Haasdijk, E., Smit, S.K., Eiben, A.E.: Exploratory analysis of an on-line evolutionary algorithm in simulated robots. Evolutionary Intelligence 5(4), 213-230 (2012)

6. Haasdijk, E., Weel, B., Eiben, A.E.: Right on the MONEE. In: Blum, C. (ed.) Proceedings of GECCO '13. pp. 207-214. ACM Press (2013)

7. Hart, E., Steyven, A., Paechter, B.: Improving survivability in environment-driven distributed evolutionary algorithms through explicit relative fitness and fitness proportionate communication. In: Silva, S. (ed.) Proceedings of GECCO '15. pp. 169-176. ACM Press (2015)

8. Heinerman, J., Rango, M., Eiben, A.E.: Evolution, individual learning, and social learning in a swarm of real robots. In: 2015 IEEE Symposium Series on Computational Intelligence. pp. 1055-1062. IEEE (2015)

9. Steyven, A.: Interactive 3D model of mEDEA_rf parameter sweep fitness landscape (2016), http://research.steyven.de/conf/ppsn2016/

10. Steyven, A., Hart, E., Paechter, B.: The cost of communication. In: Silva, S. (ed.) GECCO Companion '15. pp. 1239-1240. ACM Press (2015) 